Mathematical Modelling and Analysis

Volume 19 Number 5, November 2014, 607-626

http://dx.doi.org/10.3846/13926292.2014.979334

(C) Vilnius Gediminas Technical University, 2014
Publisher: Taylor\&Francis and VGTU

http://www.tandfonline.com/TMMA

Print ISSN: 1392-6292

Online ISSN: 1648-3510

\title{
A Viscoelastic Contact Problem with Adhesion and Surface Memory Effects*
}

\author{
Mircea Sofonea ${ }^{a}$ and Flavius Pătrulescu ${ }^{b, c}$ \\ ${ }^{a}$ Laboratoire de Mathématiques et Physique, Université de Perpignan Via \\ Domitia \\ 52 Avenue de Paul Alduy, 66860 Perpignan, France \\ ${ }^{b}$ Tiberiu Popoviciu Institute of Numerical Analysis, Romanian Academy \\ P.O. Box 68-1, 400110 Cluj-Napoca, Romania \\ ${ }^{c}$ Faculty of Mathematics and Computer Science, Babeş-Bolyai University \\ Kogălniceanu street, no. 1, 400084, Cluj-Napoca, Romania \\ E-mail(corresp.): sofonea@univ-perp.fr \\ E-mail: fpatrulescu@ictp.acad.ro
}

Received March 21, 2014; revised October 3, 2014; published online November 1, 2014

\begin{abstract}
We consider a mathematical model which describes the quasistatic contact between a viscoelastic body and an obstacle, the so-called foundation. The material's behavior is modelled with a constitutive law with long memory. The contact is with normal compliance, unilateral constraint, memory effects and adhesion. We present the classical formulation of the problem, then we derive its variational formulation and prove an existence and uniqueness result. The proof is based on arguments of variational inequalities and fixed point.
\end{abstract}

Keywords: existence, fixed point, mathematical model.

AMS Subject Classification: 74M15; 74G25; 74G30; 74D05; 49J40.

\section{Introduction}

Processes of contact with adhesion are important in many industrial settings where parts, usually nonmetallic, are glued together. For this reason, a considerable effort has been made in their modeling, analysis, numerical analysis, and numerical simulations and, as a result, the engineering and computational literature on this related topics is extensive. Moreover, the mathematical literature devoted to the analysis of adhesive contact process is rapidly growing.

General models with adhesion can be found in $[7,8,9,15,18]$ and the references therein. In particular, a description of the derivation of various equations

* The work of the second author has been partially supported by project No. POSDRU/159/1.5/S/132400 at Babeş-Bolyai University. 
and conditions related to the adhesive contact can be found in [9]. The analysis of various contact models with adhesion, including existence and uniqueness results for weak solutions, can be found in $[1,2,3,4,20,25,26]$, for instance. In carrying out this analysis, a systematic use of results on elliptic and evolutionary variational inequalities, convex analysis, nonlinear equations with monotone operators, and fixed points of operators was made. The numerical analysis of quasistatic and dynamic models of adhesive contact can be found in [20]. There, fully discrete schemes were considered and error estimates were derived. Moreover, an application of the theory in the medical field of prosthetic limbs was described in $[16,17]$. There, the bonding arises between the artificial limb and the tissue and is of considerable importance, since debonding may lead to decrease in the person's ability to use the limb. The main ingredient in the models presented in all the above mentioned papers is the introduction of a surface internal variable, the bonding field, which describes the fractional density of active bonds on the contact surface. As a fraction its values are restricted to $0 \leq \beta \leq 1$. When $\beta=1$ at a point of the contact surface, the adhesion is complete and all the bonds are active; when $\beta=0$ all the bonds are inactive, severed, and there is no adhesion; finally, when $0<\beta<1$ the adhesion is partial and only a fraction $\beta$ of the bonds is active.

In this paper, we cover the modelling and variational analysis of a new contact problem with adhesion within the infinitesimal strain theory. The evolution of the bonding field is described by a general first order ordinary differential equation, already used in the previously cited papers. Nevertheless, we introduce three novelties in the contact model, which make the difference with our previous papers. First, we describe material's behavior by a viscoelastic constitutive law with long memory. Second, we model the adhesive contact with a normal compliance condition with unilateral condition which takes into account the memory effect of the surfaces. A similar condition was introduced in $[23,24]$ in the study of frictionless contact process without adhesion. Also, a contact condition with normal compliance, unilateral constraint and adhesion was used in [11]. There, in contrast with this paper, the memory effects of the foundation were neglected, the process was assumed to be dynamic and the material's behavior was described with an elastic-visco-plastic constitutive law. The third novelty arises in the fact that, unlike a large number of references, the adhesive contact problem considered in this paper are formulated on the unbounded interval of time $\mathbb{R}_{+}=[0, \infty)$. This implies the use of the framework of Fréchet spaces of continuous functions, instead of that of the classical Banach spaces of continuous functions defined on a bounded interval of time, used in our previous papers.

The rest of the paper is organized as follows. In Section 2 we introduce some notations and preliminary material. In Section 3 we provide a detailed description of the model of adhesive contact. Then, in Section 4, we list the assumptions on the data, derive the variational formulation of the problem and state our main existence and uniqueness result, Theorem 2. The proof is provided in Section 5. It is based on arguments of variational inequalities and fixed point. 


\section{Notations and Preliminaries}

In this short section we present the notations we shall use and some preliminary material. For further details we refer the reader to $[10,18,22]$. Everywhere in this paper we use the notation $\mathbb{N}$ for the set of positive integers and $\mathbb{R}_{+}$will represent the set of nonnegative real numbers, i.e. $\mathbb{R}_{+}=[0,+\infty)$. For a given $r \in \mathbb{R}$ we denote by $r^{+}$its positive part, i.e. $r^{+}=\max \{r, 0\}$. Let $d \in \mathbb{N}$. Then, we denote by $\mathbb{S}^{d}$ the space of second order symmetric tensors on $\mathbb{R}^{d}$. The inner product and norm on $\mathbb{R}^{d}$ and $\mathbb{S}^{d}$ are defined by

$$
\begin{array}{ccc}
\boldsymbol{u} \cdot \boldsymbol{v}=u_{i} v_{i}, & \|\boldsymbol{v}\|=(\boldsymbol{v} \cdot \boldsymbol{v})^{\frac{1}{2}} & \forall \boldsymbol{u}, \boldsymbol{v} \in \mathbb{R}^{d}, \\
\boldsymbol{\sigma} \cdot \boldsymbol{\tau}=\sigma_{i j} \tau_{i j}, & \|\boldsymbol{\tau}\|=(\boldsymbol{\tau} \cdot \boldsymbol{\tau})^{\frac{1}{2}} & \forall \boldsymbol{\sigma}, \boldsymbol{\tau} \in \mathbb{S}^{d} .
\end{array}
$$

Here and below the indices $i, j, k, l$ run between 1 and $d$ and, unless stated otherwise, the summation convention over repeated indices is used.

Let $\Omega$ be a bounded domain $\Omega \subset \mathbb{R}^{d}(d=1,2,3)$ with a Lipschitz continuous boundary $\Gamma$ and let $\Gamma_{1}$ be a measurable part of $\Gamma$ such that meas $\left(\Gamma_{1}\right)>0$. We use the notation $\boldsymbol{x}=\left(x_{i}\right)$ for a typical point in $\Omega \cup \Gamma$ and we denote by $\boldsymbol{\nu}=\left(\nu_{i}\right)$ the outward unit normal at $\Gamma$. Also, an index that follows a comma represents the partial derivative with respect to the corresponding component of the spatial variable, e.g. $u_{i, j}=\partial u_{i} / \partial x_{j}$. We use standard notations for the Lebesgue and Sobolev spaces associated to $\Omega$ and $\Gamma$ and, moreover, we consider the spaces

$$
\begin{aligned}
& V=\left\{\boldsymbol{v}=\left(v_{i}\right) \in H^{1}(\Omega)^{d}: \boldsymbol{v}=\mathbf{0} \text { on } \Gamma_{1}\right\}, \\
& Q=\left\{\boldsymbol{\tau}=\left(\tau_{i j}\right) \in L^{2}(\Omega)^{d \times d}: \tau_{i j}=\tau_{j i}\right\} .
\end{aligned}
$$

These are real Hilbert spaces endowed with the inner products

$$
(\boldsymbol{u}, \boldsymbol{v})_{V}=\int_{\Omega} \varepsilon(\boldsymbol{u}) \cdot \varepsilon(\boldsymbol{v}) d x, \quad(\boldsymbol{\sigma}, \boldsymbol{\tau})_{Q}=\int_{\Omega} \boldsymbol{\sigma} \cdot \boldsymbol{\tau} d x
$$

and the associated norms $\|\cdot\|_{V}$ and $\|\cdot\|_{Q}$, respectively. Here $\varepsilon$ represents the deformation operator given by

$$
\boldsymbol{\varepsilon}(\boldsymbol{v})=\left(\varepsilon_{i j}(\boldsymbol{v})\right), \quad \varepsilon_{i j}(\boldsymbol{v})=\frac{1}{2}\left(v_{i, j}+v_{j, i}\right) \forall \boldsymbol{v} \in H^{1}(\Omega)^{d} .
$$

Completeness of the space $\left(V,\|\cdot\|_{V}\right)$ follows from the assumption meas $\left(\Gamma_{1}\right)>0$, which allows the use of Korn's inequality.

For an element $\boldsymbol{v} \in V$ we still write $\boldsymbol{v}$ for the trace of $\boldsymbol{v}$ on the boundary $\Gamma$. We denote by $v_{\nu}$ and $\boldsymbol{v}_{\tau}$ the normal and the tangential component of $\boldsymbol{v}$ on $\Gamma$, respectively, defined by $v_{\nu}=\boldsymbol{v} \cdot \boldsymbol{\nu}, \boldsymbol{v}_{\tau}=\boldsymbol{v}-v_{\nu} \boldsymbol{\nu}$. Let $\Gamma_{3}$ be a measurable part of $\Gamma$. Then, by the Sobolev trace theorem, there exists a positive constant $c_{0}$ which depends on $\Omega, \Gamma_{1}$ and $\Gamma_{3}$ such that

$$
\|\boldsymbol{v}\|_{L^{2}\left(\Gamma_{3}\right)^{d}} \leq c_{0}\|\boldsymbol{v}\|_{V} \quad \forall \boldsymbol{v} \in V .
$$

For a regular function $\boldsymbol{\sigma}: \Omega \cup \Gamma \rightarrow \mathbb{S}^{d}$ we denote by $\sigma_{\nu}$ and $\boldsymbol{\sigma}_{\tau}$ the normal and the tangential components of the vector $\boldsymbol{\sigma} \boldsymbol{\nu}$ on $\Gamma$, respectively, and we recall that $\sigma_{\nu}=\boldsymbol{\sigma} \boldsymbol{\nu} \cdot \boldsymbol{\nu}$ and $\boldsymbol{\sigma}_{\tau}=\boldsymbol{\sigma} \boldsymbol{\nu}-\sigma_{\nu} \boldsymbol{\nu}$. 
We also introduce the space of fourth order tensor fields given by

$$
\mathbf{Q}_{\infty}=\left\{\mathcal{E}=\left(\mathcal{E}_{i j k l}\right) \mid \mathcal{E}_{i j k l}=\mathcal{E}_{j i k l}=\mathcal{E}_{k l i j} \in L^{\infty}(\Omega), 1 \leq i, j, k, l \leq d\right\}
$$

and we recall that $\mathbf{Q}_{\infty}$ is a real Banach space with the norm

$$
\|\mathcal{E}\|_{\mathbf{Q}_{\infty}}=\sum_{1 \leq i, j, k, l \leq d}\left\|\mathcal{E}_{i j k l}\right\|_{L^{\infty}(\Omega)}
$$

Moreover, a simple calculation shows that

$$
\|\mathcal{E} \boldsymbol{\tau}\|_{Q} \leq\|\mathcal{E}\|_{\mathbf{Q}_{\infty}}\|\boldsymbol{\tau}\|_{Q} \quad \forall \mathcal{E} \in \mathbf{Q}_{\infty}, \boldsymbol{\tau} \in Q
$$

Given a normed space $\left(X,\|\cdot\|_{X}\right)$ we use the notation $C\left(\mathbb{R}_{+} ; X\right)$ for the space of continuous functions defined on $\mathbb{R}_{+}$with values in $X$. It is well known that, if $X$ is a Banach space, then $C\left(\mathbb{R}_{+} ; X\right)$ can be organized in a canonical way as a Fréchet space, i.e., as a complete metric space in which the corresponding topology is induced by a countable family of seminorms. Details can be found in [5] and [14], for instance. Also, for a subset $K \subset X$ we still use the symbol $C\left(\mathbb{R}_{+} ; K\right)$ for the set of continuous functions defined on $\mathbb{R}_{+}$with values in $K$. Finally, for $n \in \mathbb{N}$ and $K \subset X$ we denote by $C([0, n] ; K)$ the set of continuous functions defined on $[0, n]$ with values in $K$.

We end this section with the following result which will be used in Section 5 of the paper.

Theorem 1. Let $\left(X,\|\cdot\|_{X}\right)$ be a Banach space, $K$ a nonempty closed subset of $X$ and let $\Lambda: C\left(\mathbb{R}_{+} ; K\right) \rightarrow C\left(\mathbb{R}_{+} ; K\right)$ be a nonlinear operator. Assume that there exists $k \in \mathbb{N}$ with the following property: for all $n \in \mathbb{N}$ there exist two constants $c_{n} \geq 0$ and $d_{n} \in[0,1)$ such that

$$
\|\Lambda u(t)-\Lambda v(t)\|_{X}^{k} \leq c_{n} \int_{0}^{t}\|u(s)-v(s)\|_{X}^{k} d s+d_{n}\|u(t)-v(t)\|_{X}^{k}
$$

for all $u, v \in C\left(\mathbb{R}_{+} ; K\right)$ and for all $t \in[0, n]$. Then the operator $\Lambda$ has a unique fixed point $\eta^{*} \in C\left(\mathbb{R}_{+} ; K\right)$.

Theorem 1 was proved in [19] in the case when $K=X$. Nevertheless, a careful examination of the proof shows that the theorem is still valid for operators $\Lambda: C\left(\mathbb{R}_{+} ; K\right) \rightarrow C\left(\mathbb{R}_{+} ; K\right)$, provided that $K$ is a nonempty closed part of $X$. The modification in proof are straightforward and, therefore, we do not provide the details. We only mention that the difference consists in the use of the Banach fixed point argument for contractive maps defined on the set $C([0, n] ; K)$ with values on $C([0, n] ; K)$, for all $n \in \mathbb{N}$, instead of contractive maps defined on the space $C([0, T] ; X)$ with values in $C([0, T] ; X)$.

\section{Problem Statement}

The physical setting is as follows. A viscoelastic body occupies a bounded domain $\Omega \subset \mathbb{R}^{d}(d=1,2,3)$ with a Lipschitz continuous boundary $\Gamma$, divided into three measurable parts $\Gamma_{1}, \Gamma_{2}$ and $\Gamma_{3}$, such that meas $\left(\Gamma_{1}\right)>0$. The body 
is subject to the action of body forces of density $f_{0}$, is fixed on $\Gamma_{1}$ and is submitted to the action of surface tractions of density $\boldsymbol{f}_{2}$ on $\Gamma_{2}$. Moreover, the body is in adhesive contact on $\Gamma_{3}$ with an obstacle, the so-called foundation. We adopt the framework of the small strain theory, we assume that the contact process is quasistatic and we study it in the interval of time $\mathbb{R}_{+}=[0, \infty)$. To derive a mathematical model which corresponds to this physical setting we need to precise the constitutive law of the material, the balance equations and the boundary conditions, as well.

In this paper we assume that the material's behavior follows a viscoelastic constitutive law with long memory of the form

$$
\boldsymbol{\sigma}(t)=\mathcal{A} \boldsymbol{\varepsilon}(\boldsymbol{u}(t))+\int_{0}^{t} \mathcal{B}(t-s) \boldsymbol{\varepsilon}(\boldsymbol{u}(s)) d s \quad \text { in } \Omega,
$$

where, here and below, $\boldsymbol{u}$ denotes the displacement field, $\boldsymbol{\sigma}$ represents the stress field, $\boldsymbol{\varepsilon}(\boldsymbol{u})$ is the linearized strain tensor and $t \in \mathbb{R}_{+}$represents the time variable. Also, $\mathcal{A}$ and $\mathcal{B}$ represent the elasticity operator and the relaxation tensor, respectively, and are assumed to verify the following conditions:

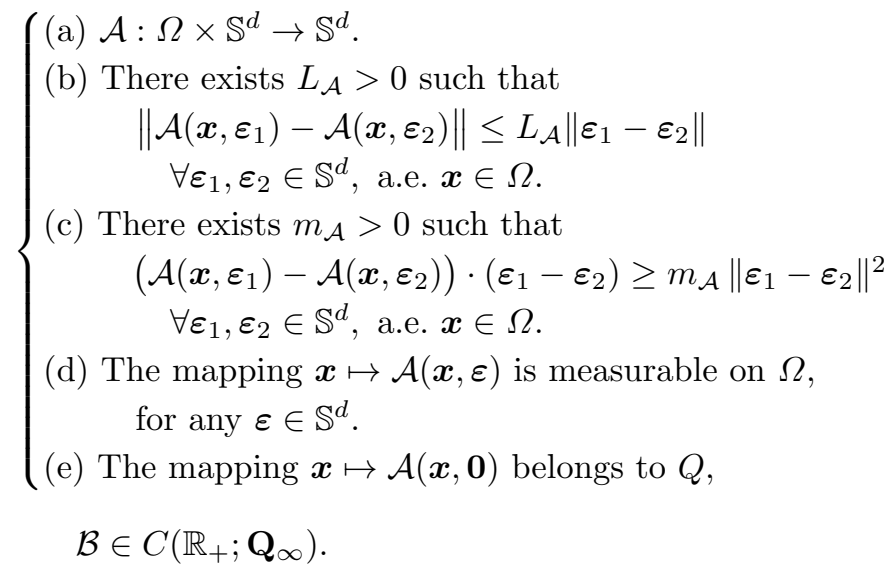

Note that in (3.1) and below, in order to simplify the notation, we do not indicate explicitly the dependence of various functions on the spatial variable $\boldsymbol{x}$. Various examples and mechanical interpretation concerning viscoelastic constitutive laws of the from $(3.1)$ can be found in $[6,21,22]$.

Next, since process is quasistatic, we shall use the equilibrium equation

$$
\operatorname{Div} \boldsymbol{\sigma}(t)+\boldsymbol{f}_{0}(t)=\mathbf{0} \quad \text { in } \Omega,
$$

where Div denotes the divergence operator for tensor valued functions, i.e. $\operatorname{Div} \boldsymbol{\sigma}=\left(\sigma_{i j, j}\right)$. This equation shows that at each time moment the external forces are balanced by the internal stresses. Moreover, since the body is fixed on $\Gamma_{1}$ and given tractions are acting on $\Gamma_{2}$ we impose the following displacement-traction conditions:

$$
\boldsymbol{u}(t)=\mathbf{0} \quad \text { on } \Gamma_{1}, \quad \boldsymbol{\sigma}(t) \boldsymbol{\nu}=\boldsymbol{f}_{2}(t) \quad \text { on } \Gamma_{2} .
$$


In addition, we assume that the densities of body forces and surface tractions have regularity

$$
\boldsymbol{f}_{0} \in C\left(\mathbb{R}_{+} ; L^{2}(\Omega)^{d}\right), \quad \boldsymbol{f}_{2} \in C\left(\mathbb{R}_{+} ; L^{2}\left(\Gamma_{2}\right)^{d}\right) .
$$

We now turn to the description on the adhesive contact conditions on the surface $\Gamma_{3}$ in which our main interest is. First, we assume that the penetration is limited by a bound $g>0$ and, therefore, at each time moment $t \in \mathbb{R}_{+}$, the normal displacement satisfies the inequality

$$
u_{\nu}(t) \leq g \text { on } \Gamma_{3}
$$

Next, we assume that the normal stress has an additive decomposition of the form

$$
\sigma_{\nu}(t)=\sigma_{\nu}^{D}(t)+\sigma_{\nu}^{R}(t)+\sigma_{\nu}^{M}(t)+\sigma_{\nu}^{A}(t) \quad \text { on } \Gamma_{3},
$$

where the functions $\sigma_{\nu}^{D}(t), \sigma_{\nu}^{R}(t), \sigma_{\nu}^{A}(t)$ and $\sigma_{\nu}^{M}(t)$ describe the deformability, the rigidity, the adhesive and the surface memory properties of the foundation. We assume that $\sigma_{\nu}^{D}(t)$ satisfies the normal compliance contact condition:

$$
-\sigma_{\nu}^{D}(t)=p_{\nu}\left(u_{\nu}(t)\right) \quad \text { on } \Gamma_{3}
$$

Here $p_{\nu}$ is a given function which satisfies

$$
\left\{\begin{array}{l}
\text { (a) } p_{\nu}: \Gamma_{3} \times \mathbb{R} \rightarrow \mathbb{R}_{+} . \\
\text {(b) There exists } L_{\nu}>0 \text { such that } \\
\left|p_{\nu}\left(\boldsymbol{x}, r_{1}\right)-p_{\nu}\left(\boldsymbol{x}, r_{2}\right)\right| \leq L_{\nu}\left|r_{1}-r_{2}\right| \\
\forall r_{1}, r_{2} \in \mathbb{R}, \text { a.e. } \boldsymbol{x} \in \Gamma_{3} . \\
\text { (c) }\left(p_{\nu}\left(\boldsymbol{x}, r_{1}\right)-p_{\nu}\left(\boldsymbol{x}, r_{2}\right)\right)\left(r_{1}-r_{2}\right) \geq 0 \\
\forall r_{1}, r_{2} \in \mathbb{R}, \text { a.e. } \boldsymbol{x} \in \Gamma_{3} . \\
\text { (d) The mapping } \boldsymbol{x} \mapsto p_{\nu}(\boldsymbol{x}, r) \text { is measurable on } \Gamma_{3}, \\
\text { for any } r \in \mathbb{R} . \\
\text { (e) } p_{\nu}(\boldsymbol{x}, r)=0 \text { for all } r \leq 0, \text { a.e. } \boldsymbol{x} \in \Gamma_{3} .
\end{array}\right.
$$

The part $\sigma_{\nu}^{R}(t)$ of the normal stress satisfies the Signorini condition in the form with a gap function, i.e.

$$
\sigma_{\nu}^{R}(t) \leq 0, \quad \sigma_{\nu}^{R}(t)\left(u_{\nu}(t)-g\right)=0 \quad \text { on } \Gamma_{3} .
$$

Details on the Signorini condition and normal compliance function can be found in $[10,12,13,18]$, for instance. Here we restrict ourselves to recall that the normal compliance condition describes the contact with a deformable foundation and the Signorini contact condition describes the contact with a perfectly rigid foundation.

The function $\sigma_{\nu}^{M}(t)$ satisfies the condition

$$
\left\{\begin{array}{l}
\left|\sigma_{\nu}^{M}(t)\right| \leq \int_{0}^{t} b(t-s) u_{\nu}^{+}(s) d s \\
\sigma_{\nu}^{M}(t)=0 \quad \text { if } u_{\nu}(t)<0, \\
\sigma_{\nu}^{M}(t)=-\int_{0}^{t} b(t-s) u_{\nu}^{+}(s) d s \quad \text { if } u_{\nu}(t)>0
\end{array} \quad \text { on } \Gamma_{3}\right.
$$


where $b$ is a surface memory function which verifies

$$
b \in C\left(\mathbb{R}_{+} ; L^{\infty}\left(\Gamma_{3}\right)\right), \quad b(t, \boldsymbol{x}) \geq 0 \quad \text { for all } t \in \mathbb{R}_{+} \text {, a.e. } \boldsymbol{x} \in \Gamma_{3} .
$$

Details on this condition can be found in [24]. Finally, the contribution of the bonding to the normal traction, $\sigma_{\nu}^{A}(t)$, satisfies

$$
\sigma_{\nu}^{A}(t)=\gamma_{\nu} \beta^{2}(t) \widetilde{R}\left(u_{\nu}(t)\right) \quad \text { on } \Gamma_{3},
$$

where $\widetilde{R}$ is the truncation function given by

$$
\widetilde{R}(s)= \begin{cases}L & \text { if } s<-L, \\ -s & \text { if }-L \leq s \leq 0 \\ 0 & \text { if } s>0\end{cases}
$$

Here and below $L>0$ is the characteristic length of the bond, beyond which it stretches without offering any additional resistance (see, e.g., [15]) and $\gamma_{\nu}$ represents an adhesion coefficient. More details on this condition can be found in $[20]$.

We combine (3.7), (3.8), (3.13) and denote $-\sigma_{\nu}^{M}(t)=\xi(t)$ to see that

$$
\sigma_{\nu}^{R}(t)=\sigma_{\nu}(t)+p_{\nu}\left(u_{\nu}(t)\right)-\gamma_{\nu} \beta^{2}(t) \widetilde{R}\left(u_{\nu}(t)\right)+\xi(t) \quad \text { on } \Gamma_{3} .
$$

Then we substitute equality (3.15) in (3.10) and use (3.6), (3.11) and (3.12) to obtain the following contact condition

$$
\begin{cases}u_{\nu}(t) \leq g, \quad \sigma_{\nu}(t)+p_{\nu}\left(u_{\nu}(t)\right)+\xi(t)-\gamma_{\nu} \beta^{2}(t) \widetilde{R}\left(u_{\nu}(t)\right) \leq 0, & \\ \left(u_{\nu}(t)-g\right)\left(\sigma_{\nu}(t)+p_{\nu}\left(u_{\nu}(t)\right)+\xi(t)-\gamma_{\nu} \beta^{2}(t) \widetilde{R}\left(u_{\nu}(t)\right)\right)=0, & \text { on } \Gamma_{3} . \\ 0 \leq \xi(t) \leq \int_{0}^{t} b(t-s) u_{\nu}^{+}(s) d s, & \\ \xi(t)=0 \quad \text { if } u_{\nu}(t)<0, & \text { if } u_{\nu}(t)>0,\end{cases}
$$

To complete our model we assume that the resistance to tangential motion is generated mainly by the glue, and the frictional traction can be neglected. In particular, when all the adhesive bonds are inactive, or broken, the motion is frictionless. Thus, the tangential traction depends on the intensity of adhesion and on the tangential displacement, but only up to the bond length $L$, that is

$$
-\boldsymbol{\sigma}_{\tau}(t)=p_{\tau}(\beta(t)) \boldsymbol{R}^{*}\left(\boldsymbol{u}_{\tau}(t)\right) \text { on } \Gamma_{3},
$$

where the truncation operator $\boldsymbol{R}^{*}$ is defined by

$$
\boldsymbol{R}^{*}(\boldsymbol{v})= \begin{cases}\boldsymbol{v} & \text { if }\|\boldsymbol{v}\| \leq L, \\ \frac{L}{\|\boldsymbol{v}\|} \boldsymbol{v} & \text { if }\|\boldsymbol{v}\| \geq L .\end{cases}
$$


Then, $p_{\tau}(\beta)$ acts as the stiffness or spring constant, and the traction is in direction opposite to the displacement. The tangential function $p_{\tau}$ satisfies

$$
\left\{\begin{array}{l}
\text { (a) } p_{\tau}: \Gamma_{3} \times \mathbb{R} \rightarrow \mathbb{R}_{+} \cdot \\
\text { (b) There exists } L_{\tau}>0 \text { such that } \\
\quad\left|p_{\tau}\left(\boldsymbol{x}, \beta_{1}\right)-p_{\tau}\left(\boldsymbol{x}, \beta_{2}\right)\right| \leq L_{\tau}\left|\beta_{1}-\beta_{2}\right| \\
\forall \beta_{1}, \beta_{2} \in \mathbb{R}, \text { a.e. } \boldsymbol{x} \in \Gamma_{3} . \\
\text { (c) There exists } M_{\tau}>0 \text { such that } p_{\tau}(\boldsymbol{x}, \beta) \leq M_{\tau} \\
\forall \beta \in \mathbb{R}, \text { a.e. } \boldsymbol{x} \in \Gamma_{3} . \\
\text { (d) The mapping } \boldsymbol{x} \mapsto p_{\tau}(\boldsymbol{x}, \beta) \text { is measurable on } \Gamma_{3}, \\
\text { for any } \beta \in \mathbb{R} . \\
\text { (e) } p_{\tau}(\boldsymbol{x}, 0)=0 \text { a.e. } \boldsymbol{x} \in \Gamma_{3} .
\end{array}\right.
$$

We follow $[7,8,20]$ and assume that the bonding field satisfies the unilateral constraint

$$
0 \leq \beta(t) \leq 1 \text { on } \Gamma_{3}
$$

Moreover, its evolution is governed by the differential equation

$$
\dot{\beta}(t)=-\left(\gamma_{\nu} \beta(t)\left[R\left(u_{\nu}(t)\right)\right]^{2}-\varepsilon_{a}\right)^{+} \text {on } \Gamma_{3},
$$

in which $\varepsilon_{a}$ represents the Dupré energy and $R$ is the truncation operator given by

$$
R(s)= \begin{cases}-L & \text { if } s<-L \\ s & \text { if }-L \leq s \leq L \\ L & \text { if } s>L\end{cases}
$$

We complete this differential equation with the initial condition

$$
\beta(0)=\beta_{0} \text { on } \Gamma_{3}
$$

and we assume that the adhesion coefficient, $\gamma_{\nu}$, the Dupré energy $\varepsilon_{a}$, and initial bonding field, $\beta_{0}$, satisfy the conditions

$$
\begin{gathered}
\gamma_{\nu} \in L^{\infty}\left(\Gamma_{3}\right), \quad \gamma_{\nu} \geq 0, \quad \varepsilon_{a} \in L^{\infty}\left(\Gamma_{3}\right), \quad \varepsilon_{a} \geq 0, \\
\beta_{0} \in L^{2}\left(\Gamma_{3}\right), \quad 0 \leq \beta_{0} \leq 1 \quad \text { a.e. on } \Gamma_{3} .
\end{gathered}
$$

We gather the above equations and conditions to obtain the following formulation of the mechanical problem of quasistatic adhesive contact with normal compliance, unilateral constraint and surface memory term.

Problem $\mathcal{P}$. Find a displacement field $\boldsymbol{u}: \Omega \times \mathbb{R}_{+} \rightarrow \mathbb{R}^{d}$, a stress field $\boldsymbol{\sigma}$ : $\Omega \times \mathbb{R}_{+} \rightarrow \mathbb{S}^{d}$ and a adhesion field $\beta: \Gamma_{3} \times \mathbb{R}_{+} \rightarrow[0,1]$ such that

$$
\begin{array}{rc}
\boldsymbol{\sigma}(t)=\mathcal{A} \varepsilon(\boldsymbol{u}(t))+\int_{0}^{t} \mathcal{B}(t-s) \boldsymbol{\varepsilon}(\boldsymbol{u}(s)) d s & \text { in } \Omega, \\
\operatorname{Div} \boldsymbol{\sigma}(t)+\boldsymbol{f}_{0}(t)=\mathbf{0} & \text { in } \Omega, \\
\boldsymbol{u}(t)=\mathbf{0} & \text { on } \Gamma_{1}, \\
\boldsymbol{\sigma}(t) \boldsymbol{\nu}=\boldsymbol{f}_{2}(t) & \text { on } \Gamma_{2}, \\
-\boldsymbol{\sigma}_{\tau}(t)=p_{\tau}(\beta(t)) \boldsymbol{R}^{*}\left(\boldsymbol{u}_{\tau}(t)\right) & \text { on } \Gamma_{3}, \\
\dot{\beta}(t)=-\left(\gamma_{\nu} \beta(t)\left[R\left(u_{\nu}(t)\right)\right]^{2}-\varepsilon_{a}\right)^{+} & \text {on } \Gamma_{3}
\end{array}
$$


for all $t \in \mathbb{R}_{+}$, there exists $\xi: \Gamma_{3} \times \mathbb{R}_{+} \rightarrow \mathbb{R}$ which satisfies

$$
\left.\begin{array}{l}
u_{\nu}(t) \leq g, \quad \sigma_{\nu}(t)+p_{\nu}\left(u_{\nu}(t)\right)+\xi(t)-\gamma_{\nu} \beta^{2}(t) \widetilde{R}\left(u_{\nu}(t)\right) \leq 0, \\
\left(u_{\nu}(t)-g\right)\left[\sigma_{\nu}(t)+p_{\nu}\left(u_{\nu}(t)\right)+\xi(t)-\gamma_{\nu} \beta^{2}(t) \widetilde{R}\left(u_{\nu}(t)\right)\right]=0, \\
0 \leq \xi(t) \leq \int_{0}^{t} b(t-s) u_{\nu}^{+}(s) d s, \\
\xi(t)=0 \quad \text { if } u_{\nu}(t)<0, \\
\xi(t)=\int_{0}^{t} b(t-s) u_{\nu}^{+}(s) d s \quad \text { if } u_{\nu}(t)>0
\end{array}\right\}
$$

for all $t \in \mathbb{R}_{+}$and, moreover,

$$
\beta(0)=\beta_{0} \quad \text { on } \Gamma_{3} .
$$

The unique weak solvability of the contact problem $\mathcal{P}$ will be stated in Section 4 and proved in Section 5. We end this current section with some additional comments of the contact condition (3.32), which represents one of the novelties of this paper.

First, we recall that (3.32) describes a condition with unilateral constraint, since inequality (3.6) holds at each time moment. The rest of the comments in this paragraph, together with the corresponding equalities and inequalities, are valid for a given point $\boldsymbol{x}$ on the contact surface $\Gamma_{3}$. Nevertheless, we recall that, for simplicity, we skip the dependence of various functions on $\boldsymbol{x}$. Assume that at a given moment $t$ there is penetration which did not reach the bound $g$, i.e. $0<u_{\nu}(t)<g$. Then, (3.32) yields

$$
-\sigma_{\nu}(t)=p_{\nu}\left(u_{\nu}(t)\right)+\int_{0}^{t} b(t-s) u_{\nu}^{+}(s) d s .
$$

This equality shows that at the moment $t$, the reaction of the foundation depends both on the current value of the penetration (represented by the term $\left.p_{\nu}\left(u_{\nu}(t)\right)\right)$ and on the history of the penetration (represented by the integral term in (3.34)). Assume now that at a given moment $t$ there is separation between the body and the foundation, i.e. $u_{\nu}(t)<0$. Then, (3.32) shows that

$$
\sigma_{\nu}(t)=\gamma_{\nu} \beta^{2}(t) \widetilde{R}\left(u_{\nu}(t)\right),
$$

i.e. the reaction of the foundation is nonnegative and depends on adhesion coefficient, on the square of intensity of adhesion and on the normal displacement, but as it does not exceed the bound length $L$. Once it exceeds it the normal traction remains constant and $\left|\sigma_{\nu}(t)\right| \leq \gamma_{\nu} L$.

In conclusion, condition (3.32) shows that delimitation takes place when there is separation. When there is penetration the contact stress is given by a normal compliance condition with memory term of the form (3.34) but up to the limit $g$. When the limit $g$ is reached, the stress is given by a Signorini-type unilateral condition. This condition can be interpreted physically as follows. The foundation is assumed to be made of a hard material covered with a thin 
layer made of a soft adhesive material with thickness $g$. The layer has adhesive viscoelastic behavior, i.e. is deformable, allows penetration and develops memory effects. The hard material is perfectly rigid and, therefore, it does not allow penetration. To summarize, the foundation has a rigid-adhesiveviscoelastic behavior; its adhesive- viscoelastic behavior is caused by the layer of the soft material while its rigid behavior is caused by the hard material.

\section{Variational Formulation and Main Result}

We now turn to the variational formulation of Problem $\mathcal{P}$ and, to this end, we assume in what follows that $(\boldsymbol{u}, \boldsymbol{\sigma}, \boldsymbol{\beta})$ represents a triple of regular functions which satisfy (3.26)-(3.33). We introduce the set of admissible displacements and the set of admissible bonding fields, respectively, defined by

$$
\begin{aligned}
& U=\left\{\boldsymbol{v} \in V: v_{\nu} \leq g \text { a.e. on } \Gamma_{3}\right\}, \\
& Z=\left\{\omega \in L^{2}\left(\Gamma_{3}\right): 0 \leq \omega \leq 1 \text { a.e. on } \Gamma_{3}\right\} .
\end{aligned}
$$

Let $\boldsymbol{v} \in U$ and $t \in \mathbb{R}_{+}$be given. We use the Green's formula to see that

$$
\int_{\Omega} \boldsymbol{\sigma}(t) \cdot \boldsymbol{\varepsilon}(\boldsymbol{v}) d x+\int_{\Omega} \operatorname{Div} \boldsymbol{\sigma}(t) \cdot \boldsymbol{v} d x=\int_{\Gamma} \boldsymbol{\sigma}(t) \boldsymbol{\nu} \cdot \boldsymbol{v} d a \quad \forall \boldsymbol{v} \in V
$$

and, combining this equality with the equilibrium equation (3.27), we find that

$$
\int_{\Omega} \boldsymbol{\sigma}(t) \cdot(\varepsilon(\boldsymbol{v})-\boldsymbol{\varepsilon}(\boldsymbol{u}(t))) d x=\int_{\Omega} \boldsymbol{f}_{0}(t) \cdot(\boldsymbol{v}-\boldsymbol{u}(t)) d x+\int_{\Gamma} \boldsymbol{\sigma}(t) \boldsymbol{\nu} \cdot(\boldsymbol{v}-\boldsymbol{u}(t)) d a .
$$

Then, we split the surface integral over $\Gamma_{1}, \Gamma_{2}$ and $\Gamma_{3}$ and, since $\boldsymbol{v}-\boldsymbol{u}(t)=\mathbf{0}$ a.e. on $\Gamma_{1}, \boldsymbol{\sigma}(t) \boldsymbol{\nu}=\boldsymbol{f}_{2}(t)$ on $\Gamma_{2}$, we deduce that

$$
\begin{gathered}
\int_{\Omega} \boldsymbol{\sigma}(t) \cdot(\varepsilon(\boldsymbol{v})-\boldsymbol{\varepsilon}(\boldsymbol{u}(t))) d x=\int_{\Omega} \boldsymbol{f}_{0}(t) \cdot(\boldsymbol{v}-\boldsymbol{u}(t)) d x \\
+\int_{\Gamma_{2}} \boldsymbol{f}_{2}(t) \cdot(\boldsymbol{v}-\boldsymbol{u}(t)) d a+\int_{\Gamma_{3}} \boldsymbol{\sigma}(t) \boldsymbol{\nu} \cdot(\boldsymbol{v}-\boldsymbol{u}(t)) d a .
\end{gathered}
$$

Moreover, since

$$
\boldsymbol{\sigma}(t) \boldsymbol{\nu} \cdot(\boldsymbol{v}-\boldsymbol{u}(t))=\sigma_{\nu}(t)\left(v_{\nu}-u_{\nu}(t)\right)+\boldsymbol{\sigma}_{\tau}(t) \cdot\left(\boldsymbol{v}_{\tau}-\boldsymbol{u}_{\tau}(t)\right) \quad \text { on } \Gamma_{3},
$$

taking into account condition (3.30) we obtain

$$
\begin{aligned}
\int_{\Omega} & \boldsymbol{\sigma}(t) \cdot(\varepsilon(\boldsymbol{v})-\boldsymbol{\varepsilon}(\boldsymbol{u}(t))) d x \\
= & \int_{\Omega} \boldsymbol{f}_{0}(t) \cdot(\boldsymbol{v}-\boldsymbol{u}(t)) d x+\int_{\Gamma_{2}} \boldsymbol{f}_{2}(t) \cdot(\boldsymbol{v}-\boldsymbol{u}(t)) d a \\
& +\int_{\Gamma_{3}} \sigma_{\nu}(t)\left(v_{\nu}-u_{\nu}(t)\right) d a-\int_{\Gamma_{3}} p_{\tau}(\beta(t)) \boldsymbol{R}^{*}\left(\boldsymbol{u}_{\tau}(t)\right) \cdot\left(\boldsymbol{v}_{\tau}-\boldsymbol{u}_{\tau}(t)\right) d a .
\end{aligned}
$$


We write now

$$
\begin{aligned}
\sigma_{\nu}(t) & \left(v_{\nu}-u_{\nu}(t)\right)=\left[\sigma_{\nu}(t)+p_{\nu}\left(u_{\nu}(t)\right)+\xi(t)-\gamma_{\nu} \beta^{2}(t) \widetilde{R}\left(u_{\nu}(t)\right)\right]\left(v_{\nu}-g\right) \\
+ & {\left[\sigma_{\nu}(t)+p_{\nu}\left(u_{\nu}(t)\right)+\xi(t)-\gamma_{\nu} \beta^{2}(t) \widetilde{R}\left(u_{\nu}(t)\right)\right]\left(g-u_{\nu}(t)\right) } \\
- & {\left[p_{\nu}\left(u_{\nu}(t)\right)+\xi(t)-\gamma_{\nu} \beta^{2}(t) \widetilde{R}\left(u_{\nu}(t)\right)\right]\left(v_{\nu}-u_{\nu}(t)\right) \text { on } \Gamma_{3}, }
\end{aligned}
$$

then we use the contact condition (3.32) and definition (4.1) of the set $U$ to see that

$$
\begin{aligned}
& \sigma_{\nu}(t)\left(v_{\nu}-u_{\nu}(t)\right) \\
& \quad \geq-\left[p_{\nu}\left(u_{\nu}(t)\right)+\xi(t)-\gamma_{\nu} \beta^{2}(t) \widetilde{R}\left(u_{\nu}(t)\right)\right]\left(v_{\nu}-u_{\nu}(t)\right) \quad \text { on } \Gamma_{3} .
\end{aligned}
$$

We use (3.32), again, and the hypothesis (3.12) on function $b$ to deduce that

$$
\left(\int_{0}^{t} b(t-s) u_{\nu}^{+}(s) d s\right)\left(v_{\nu}^{+}-u_{\nu}^{+}(t)\right) \geq \xi(t)\left(v_{\nu}-u_{\nu}(t)\right) \quad \text { on } \Gamma_{3} \text {. }
$$

Then we add the inequalities (4.4) and (4.5) and integrate the result on $\Gamma_{3}$ to find that

$$
\begin{gathered}
\int_{\Gamma_{3}} \sigma_{\nu}(t)\left(v_{\nu}-u_{\nu}(t)\right) d a+\int_{\Gamma_{3}}\left(\int_{0}^{t} b(t-s) u_{\nu}^{+}(s) d s\right)\left(v_{\nu}^{+}-u_{\nu}^{+}(t)\right) d a \\
\geq-\int_{\Gamma_{3}} p_{\nu}\left(u_{\nu}(t)\right)\left(v_{\nu}-u_{\nu}(t)\right) d a+\int_{\Gamma_{3}} \gamma_{\nu} \beta^{2}(t) \widetilde{R}\left(u_{\nu}(t)\right)\left(v_{\nu}-u_{\nu}(t)\right) d a .
\end{gathered}
$$

Finally, we combine (4.3) and (4.6) to obtain that

$$
\begin{aligned}
\int_{\Omega} \boldsymbol{\sigma}(t) \cdot\left(\boldsymbol{\varepsilon}(\boldsymbol{v})-\boldsymbol{\varepsilon}(\boldsymbol{u}(t)) d x+\int_{\Gamma_{3}} p_{\nu}\left(u_{\nu}(t)\right)\left(v_{\nu}-u_{\nu}(t)\right) d a\right. \\
\quad+\int_{\Gamma_{3}} p_{\tau}(\beta(t)) \boldsymbol{R}^{*}\left(\boldsymbol{u}_{\tau}(t)\right) \cdot\left(\boldsymbol{v}_{\tau}-\boldsymbol{u}_{\tau}(t)\right) d a \\
\quad+\int_{\Gamma_{3}}\left(\int_{0}^{t} b(t-s) u_{\nu}^{+}(s) d s\right)\left(v_{\nu}^{+}-u_{\nu}^{+}(t)\right) d a \\
\quad-\int_{\Gamma_{3}} \gamma_{\nu} \beta^{2}(t) \widetilde{R}\left(u_{\nu}(t)\right)\left(v_{\nu}-u_{\nu}(t)\right) d a \\
\geq \int_{\Omega} \boldsymbol{f}_{0}(t) \cdot(\boldsymbol{v}-\boldsymbol{u}(t)) d x+\int_{\Gamma_{2}} \boldsymbol{f}_{2}(t) \cdot(\boldsymbol{v}-\boldsymbol{u}(t)) d x \quad \forall \boldsymbol{v} \in U
\end{aligned}
$$

Next, we use the Riesz representation Theorem to define the operator $P$ : $V \rightarrow V$ and the function $\boldsymbol{f}: \mathbb{R}_{+} \rightarrow V$ by equalities

$$
\begin{aligned}
(P \boldsymbol{u}, \boldsymbol{v})_{V} & =\int_{\Gamma_{3}} p_{\nu}\left(u_{\nu}\right) v_{\nu} d a \quad \forall \boldsymbol{u}, \boldsymbol{v} \in V \\
(\boldsymbol{f}(t), \boldsymbol{v})_{V} & =\int_{\Omega} \boldsymbol{f}_{0}(t) \cdot \boldsymbol{v} d x+\int_{\Gamma_{2}} \boldsymbol{f}_{2}(t) \cdot \boldsymbol{v} d a \quad \forall \boldsymbol{v} \in V .
\end{aligned}
$$


It follows from assumptions (3.9) and (2.1) that

$$
(P \boldsymbol{u}-P \boldsymbol{v}, \boldsymbol{u}-\boldsymbol{v})_{V} \geq 0, \quad\|P \boldsymbol{u}-P \boldsymbol{v}\|_{V} \leq c_{0}^{2} L_{\nu}\|\boldsymbol{u}-\boldsymbol{v}\|_{V} \quad \forall \boldsymbol{u}, \boldsymbol{v} \in V,
$$

which shows that $P: V \rightarrow V$ is a monotone Lipschitz continuous operator. Moreover, the regularity (3.5) implies that

$$
\boldsymbol{f} \in C\left(\mathbb{R}_{+} ; V\right) .
$$

We also consider the functional $j: Z \times V \times V \rightarrow \mathbb{R}$ defined by

$$
j(\beta, \boldsymbol{u}, \boldsymbol{v})=\int_{\Gamma_{3}}\left[p_{\tau}(\beta(t)) \boldsymbol{R}^{*}\left(\boldsymbol{u}_{\tau}(t)\right) \cdot \boldsymbol{v}_{\tau}-\gamma_{\nu} \beta^{2}(t) \widetilde{R}\left(u_{\nu}(t)\right) v_{\nu}\right] d a .
$$

Then, we use (4.7) and notations (4.8)-(4.12) to deduce that

$$
\begin{aligned}
& (\boldsymbol{\sigma}(t), \boldsymbol{\varepsilon}(\boldsymbol{v})-\boldsymbol{\varepsilon}(\boldsymbol{u}(t)))_{Q}+(P \boldsymbol{u}(t), \boldsymbol{v}-\boldsymbol{u}(t))_{V} \\
& \quad+j(\beta(t), \boldsymbol{u}(t), \boldsymbol{v})-j(\beta(t), \boldsymbol{u}(t), \boldsymbol{u}(t))^{t} \\
& \quad+\left(\int_{0}^{t} b(t-s) u_{\nu}^{+}(s) d s, v_{\nu}^{+}-u_{\nu}^{+}(t)\right)_{L^{2}\left(\Gamma_{3}\right)} \geq(\boldsymbol{f}(t), \boldsymbol{v}-\boldsymbol{u}(t))_{V} .
\end{aligned}
$$

Also we integrate the differential equation (3.31) with the initial condition (3.33) to obtain that

$$
\beta(t)=\beta_{0}-\int_{0}^{t}\left(\gamma_{\nu} \beta(s)\left[R\left(u_{\nu}(s)\right)\right]^{2}-\varepsilon_{a}\right)^{+} d s \quad \text { on } \Gamma_{3} .
$$

Finally, we recall that the unilateral constraints imposed to the displacement and bonding field, combined with the definitions (4.1) and (4.2), yield

$$
\boldsymbol{u}(t) \in U, \quad \beta(t) \in Z .
$$

We now gather the constitutive law (3.26), the variational inequality (4.13), the integral equation (4.14) and the unilateral constraints (4.15) to obtain the following variational formulation of Problem $\mathcal{P}$.

Problem $\mathcal{P}^{V}$. Find a displacement field $\boldsymbol{u}: \mathbb{R}_{+} \rightarrow U$, a stress field $\boldsymbol{\sigma}: \mathbb{R}_{+} \rightarrow Q$ and a bonding field $\beta: \mathbb{R}_{+} \rightarrow Z$ such that for all $t \in \mathbb{R}_{+}$we have

$$
\begin{aligned}
\boldsymbol{\sigma}(t) & =\mathcal{A} \boldsymbol{\varepsilon}(\boldsymbol{u}(t))+\int_{0}^{t} \mathcal{B}(t-s) \boldsymbol{\varepsilon}(\boldsymbol{u}(s)) d s \\
(\boldsymbol{\sigma}(t), \boldsymbol{\varepsilon}(\boldsymbol{v})-\boldsymbol{\varepsilon}(\boldsymbol{u}(t)))_{Q}+(P \boldsymbol{u}(t), \boldsymbol{v}-\boldsymbol{u}(t))_{V} & \\
& +\left(\int_{0}^{t} b(t-s) u_{\nu}^{+}(s) d s, v_{\nu}^{+}-u_{\nu}^{+}(t)\right)_{L^{2}\left(\Gamma_{3}\right)}+j(\beta(t), \boldsymbol{u}(t), \boldsymbol{v}-\boldsymbol{u}(t)) \\
\geq & (\boldsymbol{f}(t), \boldsymbol{v}-\boldsymbol{u}(t))_{V} \quad \forall \boldsymbol{v} \in U, \\
\beta(t) & =\beta_{0}-\int_{0}^{t}\left(\gamma_{\nu} \beta(s)\left[R\left(u_{\nu}(s)\right)\right]^{2}-\varepsilon_{a}\right)^{+} d s .
\end{aligned}
$$


The existence of the unique solution of Problem $\mathcal{P}^{V}$ is stated below and proved in the next section.

Theorem 2. Assume that (3.2), (3.3), (3.5), (3.9), (3.12), (3.19), (3.24), and (3.25) hold. Then, there exists a unique solution $(\boldsymbol{u}, \boldsymbol{\sigma}, \beta)$ of Problem $\mathcal{P}^{V}$. Moreover, the solution satisfies,

$$
\boldsymbol{u} \in C\left(\mathbb{R}_{+} ; U\right), \quad \boldsymbol{\sigma} \in C\left(\mathbb{R}_{+} ; Q\right), \quad \beta \in C\left(\mathbb{R}_{+} ; Z\right) .
$$

We conclude that, under assumptions of Theorem 2, Problem $\mathcal{P}$ has a unique weak solution with regularity (4.19).

We end this section with some inequalities involving the functional $j$ which will be used in the proof of Theorem 2. Below in this section $\beta, \beta_{1}$, and $\beta_{2}$ denote elements of $Z$ while $\boldsymbol{u}_{1}, \boldsymbol{u}_{2}$, and $\boldsymbol{v}$ represent elements of $V$; recall also that $u_{i \nu}$ and $\boldsymbol{u}_{i \tau}$ denote the normal component and the tangential part of $\boldsymbol{u}_{i}$, for $i=1,2$; and, finally, $c$ denotes a generic positive constant which may depend on $\Omega, \Gamma_{1}, \Gamma_{2}, \Gamma_{3}, \mathcal{A}, \mathcal{B}, p_{\nu}, p_{\tau}, \gamma_{\nu}$ and $L$, but does not depend on $t$ nor on the rest of the input data, and whose value may change from place to place.

First, we note that $j$ is linear with respect to the last argument and, therefore,

$$
j(\beta, \boldsymbol{u},-\boldsymbol{v})=-j(\beta, \boldsymbol{u}, \boldsymbol{v}) .
$$

Next, using (4.12) we find that

$$
\begin{aligned}
j\left(\beta_{1}, \boldsymbol{u}_{1}, \boldsymbol{u}_{2}-\boldsymbol{u}_{1}\right)+j\left(\beta_{2}, \boldsymbol{u}_{2}, \boldsymbol{u}_{1}-\boldsymbol{u}_{2}\right) \\
=\int_{\Gamma_{3}} \gamma_{\nu} \beta_{1}^{2}\left(\widetilde{R}\left(u_{1 \nu}\right)-\widetilde{R}\left(u_{2 \nu}\right)\right)\left(u_{1 \nu}-u_{2 \nu}\right) d a \\
\quad+\int_{\Gamma_{3}} \gamma_{\nu}\left(\beta_{1}^{2}-\beta_{2}^{2}\right) \widetilde{R}\left(u_{2 \nu}\right)\left(u_{1 \nu}-u_{2 \nu}\right) d a \\
\quad+\int_{\Gamma_{3}} p_{\tau}\left(\beta_{1}\right)\left(\boldsymbol{R}^{*}\left(\boldsymbol{u}_{1 \tau}\right)-\boldsymbol{R}^{*}\left(\boldsymbol{u}_{2 \tau}\right)\right) \cdot\left(\boldsymbol{u}_{2 \tau}-\boldsymbol{u}_{1 \tau}\right) d a \\
\quad+\int_{\Gamma_{3}}\left(p_{\tau}\left(\beta_{1}\right)-p_{\tau}\left(\beta_{2}\right)\right) \boldsymbol{R}^{*}\left(\boldsymbol{u}_{2 \tau}\right) \cdot\left(\boldsymbol{u}_{2 \tau}-\boldsymbol{u}_{1 \tau}\right) d a
\end{aligned}
$$

and since

$$
\begin{aligned}
\left(\widetilde{R}\left(u_{1 \nu}\right)-\widetilde{R}\left(u_{2 \nu}\right)\right)\left(u_{1 \nu}-u_{2 \nu}\right) \leq 0 & \text { a.e. on } \Gamma_{3}, \\
\left(\boldsymbol{R}^{*}\left(\boldsymbol{u}_{1 \tau}\right)-\boldsymbol{R}^{*}\left(\boldsymbol{u}_{2 \tau}\right)\right) \cdot\left(\boldsymbol{u}_{2 \tau}-\boldsymbol{u}_{1 \tau}\right) \leq 0 & \text { a.e. on } \Gamma_{3},
\end{aligned}
$$

we obtain

$$
\begin{aligned}
j\left(\beta_{1}, \boldsymbol{u}_{1}, \boldsymbol{u}_{2}-\boldsymbol{u}_{1}\right)+j\left(\beta_{2}, \boldsymbol{u}_{2}, \boldsymbol{u}_{1}-\boldsymbol{u}_{2}\right) \\
\leq \int_{\Gamma_{3}} \gamma_{\nu}\left(\beta_{1}^{2}-\beta_{2}^{2}\right) \widetilde{R}\left(u_{2 \nu}\right)\left(u_{1 \nu}-u_{2 \nu}\right) d a \\
\quad+\int_{\Gamma_{3}}\left(p_{\tau}\left(\beta_{1}\right)-p_{\tau}\left(\beta_{2}\right)\right) \boldsymbol{R}^{*}\left(\boldsymbol{u}_{2 \tau}\right) \cdot\left(\boldsymbol{u}_{2 \tau}-\boldsymbol{u}_{1 \tau}\right) d a .
\end{aligned}
$$


Using now the inequalities $\left|\widetilde{R}\left(u_{2 \nu}\right)\right| \leq L,\left\|\boldsymbol{R}^{*}\left(\boldsymbol{u}_{2 \tau}\right)\right\| \leq L,\left|\beta_{1}\right| \leq 1,\left|\beta_{2}\right| \leq 1$, valid a.e. on $\Gamma_{3}$, and the property $(3.19)(\mathrm{c})$ of the function $p_{\tau}$, we deduce that

$$
j\left(\beta_{1}, \boldsymbol{u}_{1}, \boldsymbol{u}_{2}-\boldsymbol{u}_{1}\right)+j\left(\beta_{2}, \boldsymbol{u}_{2}, \boldsymbol{u}_{1}-\boldsymbol{u}_{2}\right) \leq c \int_{\Gamma_{3}}\left|\beta_{1}-\beta_{2}\right|\left\|\boldsymbol{u}_{1}-\boldsymbol{u}_{2}\right\| d a .
$$

Next, we combine the previous inequality with (2.1) to obtain

$$
j\left(\beta_{1}, \boldsymbol{u}_{1}, \boldsymbol{u}_{2}-\boldsymbol{u}_{1}\right)+j\left(\beta_{2}, \boldsymbol{u}_{2}, \boldsymbol{u}_{1}-\boldsymbol{u}_{2}\right) \leq c\left\|\beta_{1}-\beta_{2}\right\|_{L^{2}\left(\Gamma_{3}\right)}\left\|\boldsymbol{u}_{1}-\boldsymbol{u}_{2}\right\|_{V} .
$$

We now choose $\beta_{1}=\beta_{2}=\beta$ in (4.21) to find that

$$
j\left(\beta, \boldsymbol{u}_{1}, \boldsymbol{u}_{2}-\boldsymbol{u}_{1}\right)+j\left(\beta, \boldsymbol{u}_{2}, \boldsymbol{u}_{1}-\boldsymbol{u}_{2}\right) \leq 0 .
$$

Similar manipulations, based on the Lipschitz continuity of the truncation operators $\widetilde{R}$ and $\boldsymbol{R}^{*}$ and on the boundedness of the function $p_{\tau}$, show that

$$
\left|j\left(\beta, \boldsymbol{u}_{1}, \boldsymbol{v}\right)-j\left(\beta, \boldsymbol{u}_{2}, \boldsymbol{v}\right)\right| \leq c\left\|\boldsymbol{u}_{1}-\boldsymbol{u}_{2}\right\|_{V}\|\boldsymbol{v}\|_{V}
$$

Inequalities (4.21)-(4.22) and equality (4.20) will be used in various places in the next section.

\section{Proof of Theorem 2}

The proof of Theorem 2 will be carried out in several steps. To present it everywhere below we assume that the hypothesis $(3.2),(3.3),(3.5),(3.9),(3.12)$, (3.19), (3.24), and (3.25) hold. Also, we use the product space $X=Q \times L^{2}\left(\Gamma_{3}\right) \times$ $L^{2}\left(\Gamma_{3}\right)$, endowed with the norm

$$
\|\boldsymbol{\eta}\|_{X}=\|\boldsymbol{\theta}\|_{Q}+\|\zeta\|_{L^{2}\left(\Gamma_{3}\right)}+\|\beta\|_{L^{2}\left(\Gamma_{3}\right)} \quad \forall \boldsymbol{\eta}=(\boldsymbol{\theta}, \zeta, \beta) \in X .
$$

Let $\boldsymbol{\eta}=(\boldsymbol{\theta}, \zeta, \beta) \in C\left(\mathbb{R}_{+} ; Q \times L^{2}\left(\Gamma_{3}\right) \times Z\right)$. In the first step we consider the following variational problem.

Problem $\mathcal{P}_{\eta}^{V}$. Find a displacement field $\boldsymbol{u}_{\eta}: \mathbb{R}_{+} \rightarrow U$ such that, for all $t \in \mathbb{R}_{+}$, $\boldsymbol{u}_{\eta}(t) \in U$ and

$$
\begin{aligned}
& \left(\mathcal{A} \varepsilon\left(\boldsymbol{u}_{\eta}(t)\right), \boldsymbol{\varepsilon}(\boldsymbol{v})-\boldsymbol{\varepsilon}\left(\boldsymbol{u}_{\eta}(t)\right)\right)_{Q}+\left(\boldsymbol{\theta}(t), \boldsymbol{\varepsilon}(\boldsymbol{v})-\boldsymbol{\varepsilon}\left(\boldsymbol{u}_{\eta}(t)\right)\right)_{Q} \\
& \quad+\left(P \boldsymbol{u}_{\eta}(t), \boldsymbol{v}-\boldsymbol{u}_{\eta}(t)\right)_{V}+\left(\zeta^{+}(t), v_{\nu}^{+}-u_{\eta \nu}^{+}(t)\right)_{L^{2}\left(\Gamma_{3}\right)} \\
& \quad+j\left(\beta(t), \boldsymbol{u}_{\eta}(t), \boldsymbol{v}-\boldsymbol{u}_{\eta}(t)\right) \geq\left(\boldsymbol{f}(t), \boldsymbol{v}-\boldsymbol{u}_{\eta}(t)\right)_{V} \quad \forall \boldsymbol{v} \in U .
\end{aligned}
$$

We have the following result concerning this problem.

Lemma 1. There exists a unique solution to Problem $\mathcal{P}_{\eta}^{V}$ which satisfies $\boldsymbol{u}_{\eta} \in$ $C\left(\mathbb{R}_{+} ; U\right)$. Moreover, if $\boldsymbol{u}_{i}$ represents the solution of Problem $\mathcal{P}_{\eta}^{V}$ for $\boldsymbol{\eta}=\boldsymbol{\eta}_{i} \in$ $C\left(\mathbb{R}_{+} ; Q \times L^{2}\left(\Gamma_{3}\right) \times Z\right), i=1,2$, then there exists $c>0$ such that

$$
\left\|\boldsymbol{u}_{1}(t)-\boldsymbol{u}_{2}(t)\right\|_{V} \leq c\left\|\boldsymbol{\eta}_{1}(t)-\boldsymbol{\eta}_{2}(t)\right\|_{X} \quad \forall t \in \mathbb{R}_{+} .
$$


Proof. Let $t \in \mathbb{R}_{+}$and consider the operator $A_{\eta t}: V \rightarrow V$ and the functional $\varphi_{\eta t}: V \rightarrow \mathbb{R}$ defined by

$$
\begin{aligned}
\left(A_{\eta t} \boldsymbol{u}, \boldsymbol{v}\right)_{V} & =(\mathcal{A} \boldsymbol{\varepsilon}(\boldsymbol{u}), \boldsymbol{\varepsilon}(\boldsymbol{v}))_{Q}+(P \boldsymbol{u}, \boldsymbol{v})_{V}+j(\beta(t), \boldsymbol{u}, \boldsymbol{v}) \quad \forall \boldsymbol{u}, \boldsymbol{v} \in V \\
\varphi_{\eta t}(\boldsymbol{v}) & =(\boldsymbol{\theta}(t), \boldsymbol{\varepsilon}(\boldsymbol{v}))_{Q}+\left(\zeta^{+}(t), v_{\nu}^{+}\right)_{L^{2}\left(\Gamma_{3}\right)} \quad \forall \boldsymbol{v} \in V .
\end{aligned}
$$

We use $(3.2),(4.10),(4.20),(4.21)$ and $(4.22)$ to see that the operator $A_{\eta t}$ is strongly monotone and Lipschitz continuous; moreover, it is easy to see that the functional $\varphi_{\eta t}$ is convex and lower semicontinuous and, in addition, the set $U$ is a closed convex nonempty subset of $V$. Using these ingredients, it follows from standard arguments on variational inequalities (see, for instance Theorem 2.8 in [22]) that there exists a unique element $\boldsymbol{u}_{\eta t} \in U$ such that

$$
\left(A_{\eta t} \boldsymbol{u}_{\eta t}, \boldsymbol{v}-\boldsymbol{u}_{\eta t}\right)_{V}+\varphi_{\eta t}(\boldsymbol{v})-\varphi_{\eta t}\left(\boldsymbol{u}_{\eta t}\right) \geq\left(\boldsymbol{f}(t), \boldsymbol{v}-\boldsymbol{u}_{\eta t}\right)_{V} \quad \forall \boldsymbol{v} \in U .
$$

Denote $\boldsymbol{u}_{\eta}(t)=\boldsymbol{u}_{\eta t}$. Then, it follows from (5.3)-(5.5) that the element $\boldsymbol{u}_{\eta}(t) \in U$ is the unique element which solves the variational inequality (5.1).

We now prove the continuity of the function $t \mapsto \boldsymbol{u}_{\eta}(t): \mathbb{R}_{+} \rightarrow V$. To this end, let $t_{1}, t_{2} \in \mathbb{R}_{+}$and denote $\boldsymbol{u}_{\eta}\left(t_{i}\right)=\boldsymbol{u}_{i}, \boldsymbol{\theta}\left(t_{i}\right)=\boldsymbol{\theta}_{i}, \zeta\left(t_{i}\right)=\zeta_{i}, \beta\left(t_{i}\right)=\beta_{i}$, $\boldsymbol{f}\left(t_{i}\right)=\boldsymbol{f}_{i}$, for $i=1,2$. We use standard arguments in (5.1) to find that

$$
\begin{aligned}
& \left(\mathcal{A} \varepsilon\left(\boldsymbol{u}_{1}\right)-\mathcal{A} \boldsymbol{\varepsilon}\left(\boldsymbol{u}_{2}\right), \boldsymbol{\varepsilon}\left(\boldsymbol{u}_{1}\right)-\boldsymbol{\varepsilon}\left(\boldsymbol{u}_{2}\right)\right)_{Q}+\left(P \boldsymbol{u}_{1}-P \boldsymbol{u}_{2}, \boldsymbol{u}_{1}-\boldsymbol{u}_{2}\right)_{V} \\
& \quad \leq\left(\boldsymbol{\theta}_{1}-\boldsymbol{\theta}_{2}, \boldsymbol{\varepsilon}\left(\boldsymbol{u}_{2}\right)-\boldsymbol{\varepsilon}\left(\boldsymbol{u}_{1}\right)\right)_{Q}+\left(\zeta_{1}^{+}-\zeta_{2}^{+}, u_{2 \nu}^{+}-u_{1 \nu}^{+}\right)_{L^{2}\left(\Gamma_{3}\right)} \\
& \quad+j\left(\beta_{1}, \boldsymbol{u}_{1}, \boldsymbol{u}_{2}-\boldsymbol{u}_{1}\right)+j\left(\beta_{2}, \boldsymbol{u}_{2}, \boldsymbol{u}_{1}-\boldsymbol{u}_{2}\right)+\left(\boldsymbol{f}_{1}-\boldsymbol{f}_{2}, \boldsymbol{u}_{1}-\boldsymbol{u}_{2}\right)_{V} .
\end{aligned}
$$

Therefore, (3.2), (4.10), (4.21) and (2.1) yield

$$
\begin{aligned}
\left\|\boldsymbol{u}_{1}-\boldsymbol{u}_{2}\right\|_{V} \leq & c\left(\left\|\boldsymbol{\theta}_{1}-\boldsymbol{\theta}_{2}\right\|_{Q}+\left\|\zeta_{1}-\zeta_{2}\right\|_{L^{2}\left(\Gamma_{3}\right)}\right. \\
& \left.+\left\|\beta_{1}-\beta_{2}\right\|_{L^{2}\left(\Gamma_{3}\right)}+\left\|\boldsymbol{f}_{1}-\boldsymbol{f}_{2}\right\|_{V}\right)
\end{aligned}
$$

where $c$ is a positive constant. This inequality combined with (4.11) and the regularity of the functions $\boldsymbol{\theta}, \zeta, \beta$ show that $\boldsymbol{u}_{\eta} \in C\left(\mathbb{R}_{+} ; V\right)$. Thus, we conclude the existence part in Lemma 1. The uniqueness part follows from of the unique solvability of (5.1) for each $t \in \mathbb{R}_{+}$. Finally, the estimate (5.2) follows by using simuilar arguments as those used in the proof of the inequality (5.6).

We now consider the operator $\Lambda$ which maps every element $\boldsymbol{\eta}=(\boldsymbol{\theta}, \zeta, \beta) \in$ $C\left(\mathbb{R}_{+} ; Q \times L^{2}\left(\Gamma_{3}\right) \times Z\right)$ into the element $\Lambda \boldsymbol{\eta}$ given by

$$
\begin{aligned}
\Lambda \boldsymbol{\eta}(t)= & \left(\int_{0}^{t} \mathcal{B}(t-s) \boldsymbol{\varepsilon}\left(\boldsymbol{u}_{\eta}(s)\right) d s, \int_{0}^{t} b(t-s) u_{\eta \nu}^{+}(s) d s\right. \\
& \left.\left(\beta_{0}-\int_{0}^{t}\left(\gamma_{\nu} \beta(s)\left[R\left(u_{\eta \nu}(s)\right)\right]^{2}-\varepsilon_{a}\right)^{+} d s\right)^{+}\right)
\end{aligned}
$$

for all $t \in \mathbb{R}_{+}$. Here $\boldsymbol{u}_{\eta} \in C\left(\mathbb{R}_{+} ; U\right)$ is the solution of Problem $\mathcal{P}_{\eta}^{V}$ provided in Lemma 1. We have the following result. 
Lemma 2. The operator $\Lambda$ takes values in the set $C\left(\mathbb{R}_{+} ; Q \times L^{2}\left(\Gamma_{3}\right) \times Z\right)$. Moreover, it has a unique fixed point $\boldsymbol{\eta}^{*} \in C\left(\mathbb{R}_{+} ; Q \times L^{2}\left(\Gamma_{3}\right) \times Z\right)$.

Proof. Let $\boldsymbol{\eta}=(\boldsymbol{\theta}, \zeta, \beta) \in C\left(\mathbb{R}_{+} ; Q \times L^{2}\left(\Gamma_{3}\right) \times Z\right)$ and denote by $\omega$ the function defined by

$$
\omega(t)=\left(\beta_{0}-\int_{0}^{t}\left(\gamma_{\nu} \beta(s)\left[R\left(u_{\eta \nu}(s)\right)\right]^{2}-\varepsilon_{a}\right)^{+} d s\right)^{+} \quad \forall t \in \mathbb{R}_{+} .
$$

Then, using (3.24) and (3.25) it follows that $\omega(t) \in L^{2}\left(\Gamma_{3}\right)$ and, moreover, $0 \leq \omega(t) \leq \beta_{0} \leq 1$ a.e. on $\Gamma_{3}$, for all $t \in \mathbb{R}_{+}$. We conclude from here that $\omega(t) \in Z$. On the other hand, it is easy to see that $t \mapsto \Lambda(t)$ is a continuous function from $\mathbb{R}_{+}$to the product space $X=Q \times L^{2}\left(\Gamma_{3}\right) \times L^{2}\left(\Gamma_{3}\right)$, which concludes the first part of the lemma.

For the second part, we consider $\boldsymbol{\eta}_{1}=\left(\boldsymbol{\theta}_{1}, \zeta_{1}, \beta_{1}\right), \boldsymbol{\eta}_{2}=\left(\boldsymbol{\theta}_{2}, \zeta_{2}, \beta_{2}\right) \in$ $C\left(\mathbb{R}_{+} ; Q \times L^{2}\left(\Gamma_{3}\right) \times Z\right)$ and, for the sake of simplicity, we use the notation $\boldsymbol{u}_{\eta_{i}}=\boldsymbol{u}_{i}$, for $i=1,2$. Let $n \in \mathbb{N}$ and let $t \in[0, n]$. We use assumptions (3.3) and (3.12) on $\mathcal{B}$ and $b$, respectively, inequalities (2.2) and (2.1) as well as the bounds $\left|R\left(u_{1 \nu}(s)\right)\right| \leq L,\left|R\left(u_{2 \nu}(s)\right)\right| \leq L,\left|\beta_{1}(s)\right| \leq 1,\left|\beta_{2}(s)\right| \leq 1$, valid a.e. on $\Gamma_{3}$, for all $s \in[0, t]$. After some elementary calculation we deduce that

$$
\begin{aligned}
& \left\|\Lambda \boldsymbol{\eta}_{1}(t)-\Lambda \boldsymbol{\eta}_{2}(t)\right\|_{X} \leq c \max _{s \in[0, n]}\|\mathcal{B}(s)\|_{\mathbf{Q}_{\infty}} \int_{0}^{t}\left\|\boldsymbol{u}_{1}(s)-\boldsymbol{u}_{2}(s)\right\|_{V} d s \\
& \quad+c \max _{s \in[0, n]}\|b(s)\|_{L^{\infty}\left(\Gamma_{3}\right)} \int_{0}^{t}\left\|\boldsymbol{u}_{1}(s)-\boldsymbol{u}_{2}(s)\right\|_{V} d s \\
& \quad+c\left(\int_{0}^{t}\left\|\boldsymbol{u}_{1}(s)-\boldsymbol{u}_{2}(s)\right\|_{V} d s+\int_{0}^{t}\left\|\beta_{1}(s)-\beta_{2}(s)\right\|_{L^{2}\left(\Gamma_{3}\right)} d s\right),
\end{aligned}
$$

where, here and below, $c$ represent various positive constants which do not depend on $n$. We conclude from here that

$$
\begin{aligned}
& \left\|\Lambda \boldsymbol{\eta}_{1}(t)-\Lambda \boldsymbol{\eta}_{2}(t)\right\|_{X} \\
& \quad \leq c_{n} \int_{0}^{t}\left\|\boldsymbol{u}_{1}(s)-\boldsymbol{u}_{2}(s)\right\|_{V} d s+c \int_{0}^{t}\left\|\beta_{1}(s)-\beta_{2}(s)\right\|_{L^{2}\left(\Gamma_{3}\right)} d s,
\end{aligned}
$$

where now $c_{n}$ represent various positive constants which depend on $n$. We now combine the inequalities (5.10) and (5.2) to deduce that

$$
\left\|\Lambda \boldsymbol{\eta}_{1}(t)-\Lambda \boldsymbol{\eta}_{2}(t)\right\|_{X} \leq c_{n} \int_{0}^{t}\left\|\boldsymbol{\eta}_{1}(s)-\boldsymbol{\eta}_{2}(s)\right\|_{X} d s
$$

and we note that, obviously, $Q \times L^{2}\left(\Gamma_{3}\right) \times Z$ is a closed subset of the space $X$. These ingredients allow us to apply Theorem 1 to conclude the proof.

Now, we have all the ingredients needed to prove Theorem 2. 
Existence. Let $\boldsymbol{\eta}^{*}=\left(\boldsymbol{\theta}^{*}, \zeta^{*}, \beta^{*}\right) \in C\left(\mathbb{R}_{+} ; Q \times L^{2}\left(\Gamma_{3}\right) \times Z\right)$ be the fixed point of $\Lambda$ and let $\boldsymbol{u}^{*}, \boldsymbol{\sigma}^{*}$ be the functions defined by

$$
\begin{aligned}
& \boldsymbol{u}^{*}(t)=\boldsymbol{u}_{\eta^{*}}(t) \\
& \boldsymbol{\sigma}^{*}(t)=\mathcal{A} \boldsymbol{\varepsilon}\left(\boldsymbol{u}^{*}(t)\right)+\int_{0}^{t} \mathcal{B}(t-s) \boldsymbol{\varepsilon}\left(\boldsymbol{u}^{*}(s)\right) d s
\end{aligned}
$$

for all $t \in \mathbb{R}_{+}$. We recall that $\boldsymbol{\eta}^{*}=\Lambda \boldsymbol{\eta}^{*}$ and, using the equalities (5.7), (5.11) and assumption (3.12) we deduce that

$$
\begin{aligned}
\boldsymbol{\theta}^{*}(t) & =\int_{0}^{t} \mathcal{B}(t-s) \boldsymbol{\varepsilon}\left(\boldsymbol{u}^{*}(s)\right) d s \\
\zeta^{*+}(t) & =\int_{0}^{t} b(t-s) u_{\nu}^{*+}(s) d s \\
\beta^{*}(t) & =\left(\beta_{0}-\int_{0}^{t}\left(\gamma_{\nu} \beta^{*}(s)\left[R\left(u_{\nu}^{*}(s)\right)\right]^{2}-\varepsilon_{a}\right)^{+} d s\right)^{+}
\end{aligned}
$$

for all $t \in \mathbb{R}_{+}$. We show that $\left(\boldsymbol{u}^{*}, \boldsymbol{\sigma}^{*}, \beta^{*}\right)$ satisfies the system (4.16)-(4.18).

First, we note that (4.16) is a direct consequence of (5.12). Next, we write the variational inequality (5.1) for $\boldsymbol{\eta}=\boldsymbol{\eta}^{*}$ and use the equalities (5.11)-(5.14) to see that (4.17) holds. And, finally, we claim that (4.18) also holds. Indeed, let $\omega^{*}$ be the function defined by

$$
\omega^{*}(t)=\beta_{0}-\int_{0}^{t}\left(\gamma_{\nu} \beta^{*}(s)\left[R\left(u_{\nu}^{*}(s)\right)\right]^{2}-\varepsilon_{a}\right)^{+} d s \quad \forall t \in \mathbb{R}_{+} .
$$

A careful examination of equalities (5.16) and (5.15) shows that the following properties hold, a.e. on $\Gamma_{3}: t \mapsto \omega^{*}(t)$ is a non increasing function, $\omega^{*}(0)=$ $\beta_{0} \geq 0$ and, if there exists $t_{0} \in \mathbb{R}_{+}$such that $\omega^{*}\left(t_{0}\right)=0$, then $\omega^{*}(t)=0$ for all $t \geq t_{0}$. We deduce from here that $\omega^{*}(t) \geq 0$ for any $t \in \mathbb{R}_{+}$. Therefore, since (5.15) and (5.16) imply that $\beta^{*}(t)=\omega^{*}(t)^{+}$we find that

$$
\beta^{*}(t)=\omega^{*}(t) \quad \forall t \in \mathbb{R}_{+} .
$$

Equality (4.18) is now a direct consequence of the equalities (5.16) and (5.17).

This proves that the triple $\left(\boldsymbol{u}^{*}, \boldsymbol{\sigma}^{*}, \beta^{*}\right)$ represents a solution of Problem $\mathcal{P}^{V}$. The regularity expressed in (4.19) is a direct consequence of the Lemmas 1 and 2, combined with assumptions (3.2) and (3.3).

Uniqueness. The uniqueness of the solution follows from the uniqueness of the fixed point of operator $\Lambda$ defined by (5.7) combined with the unique solvability of Problem $\mathcal{P}_{\eta}^{V}$. Indeed, let $(\boldsymbol{u}, \boldsymbol{\sigma}, \beta)$ be a solution of Problem $\mathcal{P}^{V}$ which satisfies (4.19) and let $\boldsymbol{\eta}=(\boldsymbol{\theta}, \zeta, \beta) \in C\left(\mathbb{R}_{+} ; Q \times L^{2}\left(\Gamma_{3}\right) \times Z\right)$ be given by

$$
\begin{aligned}
\boldsymbol{\eta}(t)= & \left(\int_{0}^{t} \mathcal{B}(t-s) \boldsymbol{\varepsilon}(\boldsymbol{u}(s)) d s, \int_{0}^{t} b(t-s) u_{\nu}^{+}(s) d s\right. \\
& \left.\left(\beta_{0}-\int_{0}^{t}\left(\gamma_{\nu} \beta(s)\left[R\left(u_{\nu}(s)\right)\right]^{2}-\varepsilon_{a}\right)^{+} d s\right)^{+}\right)
\end{aligned}
$$


for all $t \in \mathbb{R}_{+}$. We substitute equality (4.16) in (4.17) and, using (5.18), we deduce that $\boldsymbol{u}$ satisfies the inequality (5.1), at each time moment $t \in \mathbb{R}_{+}$. On the other hand, it follows from Lemma 1 that Problem $\mathcal{P}_{\eta}^{V}$ has a unique solution, denoted $\boldsymbol{u}_{\eta}$, with regularity $\boldsymbol{u}_{\eta} \in C\left(\mathbb{R}_{+} ; U\right)$. Therefore, we conclude that

$$
\boldsymbol{u}=\boldsymbol{u}_{\eta}
$$

We use (5.19) to see that

$$
\begin{aligned}
& \int_{0}^{t} \mathcal{B}(t-s) \boldsymbol{\varepsilon}\left(\boldsymbol{u}_{\eta}(s)\right) d s=\int_{0}^{t} \mathcal{B}(t-s) \boldsymbol{\varepsilon}(\boldsymbol{u}(s)) d s \\
& \int_{0}^{t} b(t-s) u_{\eta \nu}^{+}(s) d s=\int_{0}^{t} b(t-s) u_{\nu}^{+}(s) d s \\
& \left(\beta_{0}-\int_{0}^{t}\left(\gamma_{\nu} \beta(s)\left[R\left(u_{\eta \nu}(s)\right)\right]^{2}-\varepsilon_{a}\right)^{+} d s\right)^{+} \\
& \quad=\left(\beta_{0}-\int_{0}^{t}\left(\gamma_{\nu} \beta(s)\left[R\left(u_{\nu}(s)\right)\right]^{2}-\varepsilon_{a}\right)^{+} d s\right)^{+}
\end{aligned}
$$

for all $t \in \mathbb{R}_{+}$. Therefore, (5.7) and (5.18) show that $\Lambda \boldsymbol{\eta}=\boldsymbol{\eta}$ and, using the uniqueness part in Lemma 2, we deduce that

$$
\boldsymbol{\eta}=\boldsymbol{\eta}^{*}
$$

We now use (5.19), (5.20) and (5.11) to see that

$$
\boldsymbol{u}=\boldsymbol{u}_{\eta}=\boldsymbol{u}_{\eta^{*}}=\boldsymbol{u}^{*} .
$$

Then we use (4.16), (5.21) and (5.12) to deduce that

$$
\begin{aligned}
\boldsymbol{\sigma}(t) & =\mathcal{A} \boldsymbol{\varepsilon}(\boldsymbol{u}(t))+\int_{0}^{t} \mathcal{B}(t-s) \boldsymbol{\varepsilon}(\boldsymbol{u}(s)) d s \\
& =\mathcal{A} \boldsymbol{\varepsilon}\left(\boldsymbol{u}^{*}(t)\right)+\int_{0}^{t} \mathcal{B}(t-s) \boldsymbol{\varepsilon}\left(\boldsymbol{u}^{*}(s)\right) d s=\boldsymbol{\sigma}^{*}(t) \quad \forall t \in \mathbb{R}_{+}
\end{aligned}
$$

and, finally, (5.20) and (4.18) show that

$$
\beta=\beta^{*} .
$$

The uniqueness part of the theorem is now a consequence of equalities (5.21), (5.22) and (5.23).

\section{References}

[1] E. Bonetti, G. Bonfanti and R. Rossi. Analysis of a unilateral contact problem taking into account adhesion and friction. J. Differential Equations, 253(2):438462, 2012. http://dx.doi.org/10.1016/j.jde.2012.03.017.

[2] O. Chau, J.R. Fernández, M. Shillor and M. Sofonea. Variational and numerical analysis of a quasistatic viscoelastic contact problem with adhesion. J. Comput. Appl. Math., 159:431-465, 2003.

http://dx.doi.org/10.1016/S0377-0427(03)00547-8. 
[3] O. Chau, M. Shillor and M. Sofonea. Dynamic frictionless contact with adhesion. J. Angew. Math. Phys. (ZAMP), 55:32-47, 2004. http://dx.doi.org/10.1007/s00033-003-1089-9.

[4] M. Cocu and R. Rocca. Existence results for unilateral quasistatic contact problems with friction and adhesion. Math. Model Numer. Anal., 34:981-1001, 2000. http://dx.doi.org/10.1051/m2an:2000112.

[5] C. Corduneanu. Problèmes globaux dans la théorie des équations intégrales de volterra. Ann. Math. Pure Appl., 67:349-363, 1965. http://dx.doi.org/10.1007/BF02410815.

[6] A.D. Drozdov. Finite Elasticity and Viscoelasticity-A Course in the Nonlinear Mechanics of Solids. World Scientific, Singapore, 1996.

[7] M. Frémond. Equilibre des structures qui adhèrent à leur support. C. R. Acad. Sci. Paris, 295(Série II):913-916, 1982.

[8] M. Frémond. Adhérence des solides. J. Mécanique Théorique et Appliquée, 6:383-407, 1987.

[9] M. Frémond. Non-Smooth Thermomechanics. Springer, Berlin, 2002.

[10] W. Han and M. Sofonea. Quasistatic Contact Problems in Viscoelasticity and Viscoplasticity, volume 30 of Stud. Adv. Math. American Mathematical SocietyInternational Press, 2002.

[11] J. Jarušek and M. Sofonea. On the solvability of dynamic elastic-visco-plastic contact problems with adhesion. Ann. AOSR, Ser. Math. Appl., 1:191-214, 2009 .

[12] A. Klarbring, A. Mikelič and M. Shillor. Frictional contact problems with normal compliance. Int. J. Engng. Sci., 26:811-832, 1988. http://dx.doi.org/10.1016/0020-7225(88)90032-8.

[13] A. Klarbring, A. Mikelič and M. Shillor. On friction problems with normal compliance. Nonlinear Anal., 13:935-955, 1989. http://dx.doi.org/10.1016/0362-546X(89)90022-9.

[14] J.J. Massera and J.J. Schäffer. Linear Differential Equations and Function Spaces. Academic Press, New York, London, 1966.

[15] M. Raous, L. Cangémi and M. Cocu. A consistent model coupling adhesion, friction and unilateral contact. Comput. Methods Appl. Engrg., 177:383-399, 1999. http://dx.doi.org/10.1016/S0045-7825(98)00389-2.

[16] J. Rojek and J.J. Telega. Contact problems with friction, adhesion and wear in orthopaedic biomechanics. I: General developments. J. Theor. Appl. Mech., 39:655-677, 2001.

[17] J. Rojek, J.J. Telega and S. Stupkiewicz. Contact problems with friction, adhesion and wear in orthopaedic biomechanics. II: Numerical implementation and application to implanted knee joints. J. Theor. Appl. Mech., 39:679-706, 2001.

[18] M. Shillor, M. Sofonea and J.J. Telega. Models and Analysis of Quasistatic Contact, volume 655 of Lecture Notes in Phys. Springer, Berlin, 2004.

[19] M. Sofonea, C. Avramescu and A. Matei. A fixed point result with applications in the study of viscoeplastic frictionless contact problems. Commun. Pure Appl. Anal., 7:645-658, 2008. http://dx.doi.org/10.3934/cpaa.2008.7.645.

[20] M. Sofonea, W. Han and M. Shillor. Analysis and Approximation of Contact Problems with Adhesion or Damage, volume 276 of Pure Appl. Math. ChapmanHall/ CRC Press, New York, 2006. 
[21] M. Sofonea and A. Matei. Variational Inequalities with Applications. A Study of Antiplane Frictional Contact Problems, volume 18 of Adv. Mech. Math. Springer, New York, 2009.

[22] M. Sofonea and A. Matei. Mathematical Models in Contact Mechanics, volume 398 of London Math. Soc. Lecture Note Ser. Cambridge University Press, Cambridge, 2012.

[23] M. Sofonea and F. Patrulescu. Analysis of a history-dependent frictionless contact problem. Mathematics and Mechanics of Solids, 18:409-430, 2013. http://dx.doi.org/10.1177/1081286512440004.

[24] M. Sofonea, F. Patrulescu and A. Farcaş. A viscoplastic contact problem with normal compliance, unilateral constraint and memory term. Appl. Math. Optim., 69(2):175-198, 2014. http://dx.doi.org/10.1007/s00245-013-9216-2.

[25] A. Touzaline. Study of a viscoelastic frictional contact problem with adhesion. Comment. Math. Univ. Carolin., 52(2):257-272, 2011.

[26] A. Touzaline. Analysis of a contact adhesive problem with normal compliance and nonlocal friction. Ann. Polon. Math., 104(2):175-188, 2012. http://dx.doi.org/10.4064/ap104-2-5. 